\title{
Estimation of Probabilities of Crack Detection and False Indication in Visual Inspection of Structures
}

\author{
by Yukio Fujimoto*, Member, \\ Swilem. A. M. Swilem**, Member \\ Mitsumasa Iwata*, Member
}

\begin{abstract}
Summary
The structural expressions for the distributions of the probability of crack detection (POD) and the probability of false crack indication (POF) in the visual inspection method are presented, where the scatters in the distributions are caused by many factors related to the applied inspection method and the defect conditions in the structure. In order to estimate the POD and the POF from the results of the field inspections carried out for a structure by an inspection team under a specified condition, multiple visual inspection method combined with the successive precise inspections which are carried out for limited locations is developed. The crack classification method is introduced to improve the accuracy of the capability evaluation. The applicability of the method is numerically examined for a structural model which has crack-form defects. The advantage of the method is that the mean values of POD and POF as well as their distribution properties can be estimated without using any information other than the results of field inspections.
\end{abstract}

\section{Introduction}

Non-Destructive Inspections (NDI) are carried out for the structures when initially constructed and during use to minimize risk of failure. It is recognized that NDI is not always perfect and in general the missing of defects, the indication of false defects and the erroneous measurement of crack sizes possibly occur. To grasp the capability of the inspection method is very important because the successive maintenance actions and the reliability analysis are carried out by comparing the inspection results with the capability of applied inspection method ${ }^{1,2,3)}$. This paper treats the problems related to the capability evaluation of the visual inspection method (by naked eyes or by optical tools) applied to the structures which have the crack-form defects.

POD curve ${ }^{4)}$ is usually used to assess the inspection capability and many POD curves have been obtained using various inspection methods ${ }^{5,6,7)}$. The detection probability in the POD curve is only characterized in terms of crack length which must be the important physical parameter governing the structure safety.

For an inspection method, if the detection probabilities of individual cracks are strongly correlated with crack lengths, the POD curve for the method can be widely utilized for the analyses of inspection reliability of structures. However, for visual inspections all the cracks of the same length do not have the same detec-

* Faculty of Engineering, Hiroshima University

** Graduate School. Hiroshima University tion probability. The location and the orientation of crack, the gap of crack width, the surface condition of crack and environment in which the inspection takes place all influence the chance of detection. Therefore, the detection probability itself has a scatter owing to the influences of the above factors. Although this has been pointed out by many researchers ${ }^{8}$, most of the discussions were limited to the mean value of POD and very few papers dealt with the shape of the distribution.

In this study the structural expressions for the distribution of POD and POF in the visual inspections of structures are presented, where the scatter in these distributions is caused by all factors other than crack length.

If the capability of an applied inspection method is influenced by both of the inspection condition and the crack condition in the structure, the problem arising is how to estimate the capability. The inspection capability is usually evaluated through an experiment in which representative structures with known crack lengths are inspected ${ }^{5 / 8)}$. When the estimated POD curve is applied to the analysis of inspection reliability of other structures, if either the inspection condition or the crack condition in the structure is different between experiment and the field inspection, the accuracy of the analy. sis will be lost. Ideally, the probabilities of crack detection and false indication of visual inspections should be estimated directly from the results of field inspection of the objective structures.

However, the estimations by this method are quite difficult because the true crack conditions in the structure are never informed from the results of field inspec- 
tions. A method using only detected crack data was presented to estimate the POD curve ${ }^{9)}$. However, the method is effective only when the number of true cracks and the crack length distributions can be roughly assumed according to the previous experiences. These assumption may not always hold good in practice.

In this study, an inspection procedure employing multiple visual inspection method was developed to make it possible to estimate the POD and POF properties only from the results of field inspection of the structures.

\section{POD and POF Properties of Visual Inspection Method}

\section{1 POD property}

For cracks of the same length existing in the structure, the detection probabilities by visual inspections are not always the same, while these cracks exert the same bad influence on the structural safety. The detection probability depends on the location and the orientation of crack and the feature of crack such as the shape of crack line, the gap of crack width, rust or corrosion product which covers the crack surface, the surface roughness and so on.

Fig. 1 shows the schematic sketch of the surface cracks of length $a$, in which cracks are classified into five groups from the viewpoint of detectability level. In the figure only the differences of the gap of crack width and the surface conditions are compared. This classification is carried out based on the subjective judgement of inspectors referring their inspection experiences for the similar structures. For the cracks of length $a$ in the structure to be inspected, if the probability of existence of cracks belonging to the class $i$ condition is $q_{i}$ and the detection probability of the class $i$ cracks by visual inspections is $p_{i}(a)$, then, the detection probability for all the classes of cracks of length $a$ existing in the structure is expressed by the following equation.

$$
\begin{aligned}
\operatorname{POD}(a)= & q_{1} p_{1}(a)+q_{2} p_{2}(a)+q_{3} p_{3}(a)+q_{4} p_{4}(a) \\
& +q_{5} p_{5}(a)
\end{aligned}
$$

where

$$
\begin{aligned}
& q_{1}+q_{2}+q_{3}+q_{4}+q_{5}=1.0 \\
& 1 \geqq p_{1}(a) \geqq p_{2}(a) \geqq p_{3}(a) \geqq p_{4}(a) \geqq p_{5}(a) \geqq 0.0
\end{aligned}
$$

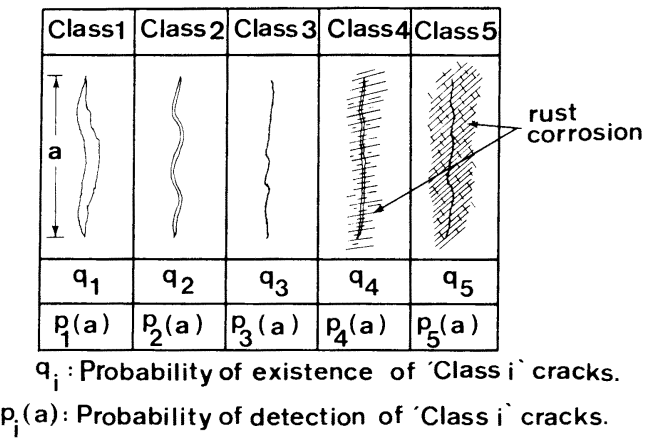

Fig. 1 Schematic sketch of surface cracks of length a .
Fig. 2(a) shows an appearance of the detection probability defined by Eq. (1). From the figure, it is obvious that $\operatorname{POD}(a)$ is the mean value of the distribution of the detection probability $p_{i}(a)$. If the classification of cracks is carried out in more detail, a continuous distribution of $p_{i}(a)$ will be obtained (see Fig. 2(b)). The shape of the distribution will be influenced by both $p_{i}(a)$ and $q_{i} . \operatorname{POD}(a)$ can still be defined as the expectation even for such a continuous distribution. Hereinafter we refer to the POD defined by Eq. (1) as the mean POD and the $p_{i}(a)$ as the POD for Class $i$ crack.

The $q_{i}$ and $p_{i}$ are interpreted in the following way.

The values of $q_{i}$ depend on the structural geometry, the feature and the cause of cracks (fatigue cracks, welding defects, etc.), the corrosion environment, the surface condition, the loading condition, etc. That is, $q_{i}$ are settled when the structure (including environmental effect) and the inspection time are specified. All the problems on structure side other than crack length are the factors which govern $q_{i}$.

The values of $p_{i}(a)$, which are the functions of crack length, depend on the inspector's ability (his experience, attitude toward the inspection), the allowed time for

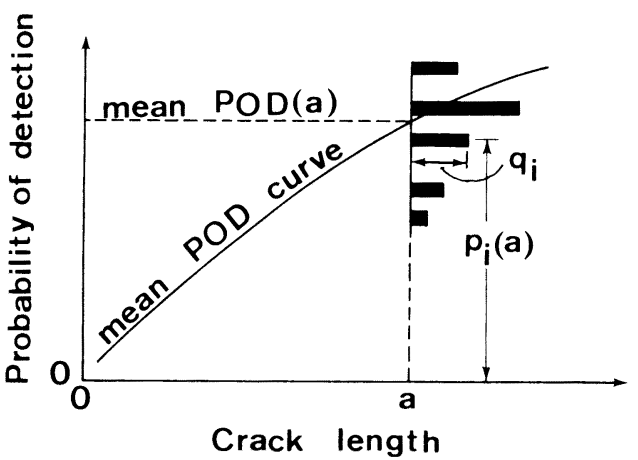

Fig. 2(a) Discrete expression of probability of crack detection.

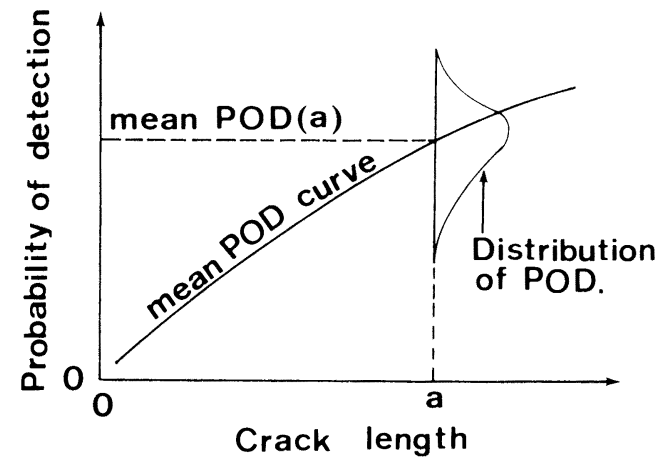

Fig. 2(b) Continuous expression of probability of crack detection. 
inspection, the performance of the inspection tool, etc. Therefore, all the problems on inspection side are the factors which govern $p_{i}(a)$.

At this point, it must be noted that, when we apply the mean POD curve obtained by inspection of a structure with known crack conditions to the inspection reliability analysis of another structure, all the conditions with respect to the inspection method and the latent cracks must be nearly the same between the two structures. It is ideal that the POD curve can be estimated through the field inspections of objective structures.

\subsection{POF property}

Mistaking the dirt or scratch for crack, the close resemblance between weld toe configuration and crack and so forth are the causes of false indications in visual inspection of structures. When all the causes of the false crack indications are classified into certain groups from the viewpoint of how the cause is prone to false indication, the POF can be expressed by the following equation.

$$
\mathrm{POF}=r_{1} s_{1}+r_{2} s_{2}+r_{3} s_{3}
$$

where

$$
\begin{aligned}
& r_{1}+r_{2}+r_{3}=1.0 \\
& 1.0 \geqq s_{1} \geqq s_{2} \geqq s_{3} \geqq 0.0
\end{aligned}
$$

In the above, false indications are classified into three groups. $r_{j}$ is the probability of existence of the causes belonging to class $j$ condition in all the causes liable to false indication and $s_{j}$ is the probability of the false crack indication due to the causes belonging to class $j$ condition. The structure of Eq. (2) is completely the same as that of Eq. (1). Therefore, the following becomes clear about the POF property of inspection.

The value of POF will be affected by both the structure to be inspected and the inspection method applied to the structure.

\section{Estimation of Visual Inspection Capability \\ Based on the Results of Field Inspection of Structures \\ In chapter 2, it was pointed out that the capability of} visual inspection should be evaluated after an objective structure and an inspection method were specified. In general, the estimation of the inspection capability becomes possible only when both the true crack distri. butions actually existing in the structure and the detected crack data are given. However the information provided by the inspection actions is limited to the detcted crack data. Therefore, some devices are necessary in the inspection process to make the estimation of the capability possible from the inspection results (records)

\section{1 Proposed inspection procedure}

The inspection procedure proposed here consists of two steps: multiple visual inspections and successive precise inspection using instrument. The inspection procedure and the relevant assumptions are summarized as follows:
(1) ' $n$ ' inspectors carry out visual inspections independently for the whole structure and report the inspection results respectively.

(2) The inspection abilities of all inspectors are assumed to be identical and the same ability is maintained throughout the inspection.

(3) There are possibilities of missing cracks and false indications in visual inspections, further the measured crack sizes are usually not accurate and sometimes uncertain.

(4) Precise inspections are carried out for the locations where one or more inspectors got the positive indications by visual inspections. It is assumed that the missing of cracks and false crack indications never occur in the precise inspections, and also the measured crack sizes are perfectly accurate.

(5) The detected cracks and the false cracks are classified into several groups from the viewpoint that to detect a crack is easy or difficult and to indicate a false crack often occurs or seldom occurs, respectively. These classifications are carried out considering all the factors other than crack length based on the subjective judgments of the inspectors.

(6) The cracks belonging to the same class are assumed to be the samples from the same statistical population. The same thing is assumed for the false cracks.

Table 1 shows an example of the inspection results (records) obtained by the above inspection procedure. Although no information is given for the cracks missed

\begin{tabular}{|c|c|c|c|c|c|}
\hline \multirow{2}{*}{ 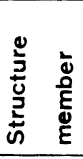 } & \multirow{2}{*}{$\begin{array}{l}\text { co } \\
\stackrel{0}{0} \\
\stackrel{0}{0}\end{array}$} & \multicolumn{2}{|c|}{$\begin{array}{c}\text { Visual } \\
\text { inspection }\end{array}$} & \multicolumn{2}{|c|}{$\begin{array}{r}\text { Precise } \\
\text { inspection }\end{array}$} \\
\hline & & {$[k / n]$} & \begin{tabular}{|l|} 
Measured \\
crack \\
length,mm
\end{tabular} & $\begin{array}{c}\text { True } \\
\text { crack } \\
\text { length,mm }\end{array}$ & $\begin{array}{c}\text { Crack } \\
\text { condition }\end{array}$ \\
\hline A & $\begin{array}{l}12 \\
25 \\
27\end{array}$ & $\begin{array}{l}2 / 3 \\
1 / 3 \\
3 / 3\end{array}$ & $\begin{array}{c}\text { about } 50 \\
\text { not clear } \\
60 \sim 70\end{array}$ & $\begin{array}{l}65 \\
\text { F.I. } \\
75\end{array}$ & 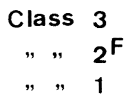 \\
\hline B & $\begin{array}{c}6 \\
9 \\
17 \\
20 \\
28\end{array}$ & $\begin{array}{l}3 / 3 \\
2 / 3 \\
1 / 3 \\
2 / 3 \\
3 / 3\end{array}$ & \begin{tabular}{|c|}
$45 \sim 55$ \\
not clear \\
$20 \sim 35$ \\
about 60 \\
$80 \sim 90$
\end{tabular} & $\begin{array}{l}60 \\
37 \\
23 \\
55 \\
80\end{array}$ & $\begin{array}{ccc}\text { Class } & 2 \\
" & " & 2 \\
" & " & 1 \\
" & " & 1 \\
" & " & 3\end{array}$ \\
\hline C & $\begin{array}{c}1 \\
5 \\
10 \\
13 \\
\end{array}$ & $\begin{array}{l}2 / 3 \\
3 / 3 \\
3 / 3 \\
1 / 3 \\
\end{array}$ & $\begin{array}{c}30 \sim 45 \\
50 \sim 60 \\
\text { about } 90 \\
25 \sim 30\end{array}$ & $\begin{array}{r}50 \\
45 \\
112 \\
\text { F.I. }\end{array}$ & $\begin{array}{ccc}\text { Class } & 2 \\
" & " & 1 \\
" & " & 3 \\
" & " & 1\end{array}$ \\
\hline & $\begin{array}{l}\vdots \\
\vdots\end{array}$ & $\vdots$ & $\begin{array}{l}\vdots \\
\vdots \\
\vdots\end{array}$ & $\vdots$ & $\vdots$ \\
\hline $\mathbf{Z}$ & $\begin{array}{c}4 \\
11 \\
16 \\
25 \\
27\end{array}$ & $\begin{array}{l}1 / 3 \\
2 / 3 \\
3 / 3 \\
1 / 3 \\
2 / 3\end{array}$ & $\begin{array}{c}\text { about } 60 \\
80 \sim 90 \\
50 \sim 65 \\
\text { not clear } \\
25 \sim 35\end{array}$ & $\begin{array}{l}64 \\
103 \\
40 \\
\text { F.I. } \\
22\end{array}$ & $\begin{array}{ccc}\text { Class } & 2 \\
" & \# & 3 \\
" & \# & 1 \\
" & \# & 2 \\
" & " & 3\end{array}$ \\
\hline
\end{tabular}
by all the inspectors in the visual inspections $(k=0)$, we

Table 1 Example of inspection results.

F.I. False indication 
can know the ratios of $(k / n)$ and the true lengths of the cracks detected by one or more inspectors $(k>0)$. Also the false cracks can be distinguished from the true cracks by these results. In the table the cracks and the false cracks are classified into three groups, and two groups, respectively.

\subsection{Estimation of $p_{i}(a)$ curves}

As the function of the detection probabilities $p_{i}(a)$ the following form was assumed ${ }^{4}$.

$$
p_{i}(a)=\frac{\exp \left\{\alpha_{i}+\beta_{i} \ln (a)\right\}}{1+\exp \left\{\alpha_{i}+\beta_{i} \ln (a)\right\}}
$$

In which $i$ means the cracks of class $i$ condition, $a$ is the crack length and $\left(\alpha_{i}, \beta_{i}\right)$ are the unknown parameters.

When $n$ inspectors with the same ability inspect a crack, if the resulting event of the crack detection is that $k$ inspectors succeed and $n-k$ fail, the probability of occurrence of the event (likelicood function) can be expressed by the following equation under the condition that at least one inspector found the crack $(k>0)$.

$$
P_{m}(k / n)=\frac{{ }_{n} C_{k} p_{i}(a)^{k}\left\{1-p_{i}(a)\right\}^{n-k}}{1-\left\{1-p_{i}(a)\right\}^{n}}
$$

The likelihood function of $\left(\alpha_{i}, \beta_{i}\right)$ for all the detected cracks belonging to class $i$ condition is given by :

$$
\boldsymbol{L}\left(\alpha_{i}, \beta_{i}\right)=\prod_{m=1}^{m_{D}} P_{m}(k / n)
$$

Where $m_{D}$ is the number of detected class $i$ cracks. Of course the value of $k$ and $a$ are different for each crack in the calculation of $p_{i}(a)$.

The most probable values of $\left(\alpha_{i}, \beta_{i}\right)$ are estimated by the Bayes' theorem ${ }^{91}$.

$$
g_{\alpha i, \beta i}(x, y)=\frac{\boldsymbol{L}\left(\alpha_{i}, \beta_{i}\right) f_{\alpha i, \beta i}(x, y)}{\sum_{x} \sum_{y}\left\{\boldsymbol{L}\left(\alpha_{i}, \beta_{i}\right) f_{\alpha i, \beta i}(x, y)\right\}}
$$

In the above equation, the prior joint density function of $\left(\alpha_{i}, \beta_{i}\right)$, denoted by $f_{\alpha i, \beta i}(x, y)$, was determined such that true values of $\left(\alpha_{i}, \beta_{i}\right)$ are included in the range of the assumed distribution. Two dimensional uniform distribution was used as prior density. The posterior joint density function of $\left(\alpha_{i}, \beta_{i}\right)$, denoted by $g_{\alpha i, \beta i}(x, y)$, was estimated by substituting Eq. ( 5 ) into Eq. (6). Then the expected values of $\left(\alpha_{i}, \beta_{i}\right)$ were calculated by performing the marginal integration for the posterior density function. Substituting the expectations into Eq. (3), the $p_{i}(a)$ curve for the class $i$ cracks can be determined.

\section{3 Estimation of probabilities $\boldsymbol{q}_{i}$}

The probabilities $q_{i}$ reveal the ratios of class $i$ cracks to all the cracks existing in the structure. This probability is determined irrespective of crack length. How ever, the value of $q_{i}$ may not be constant throughout the whole range of the crack length. For example, such event may happen that most of the long cracks belong to the high detectability class and most of the short cracks belong to the low detectability class.

Let $m_{i}$ be the number of detected class $i$ cracks whose lengths are between $a_{1}$ and $a_{2}$ (see Fig. 3). The number of class $i$ cracks actually existing in the structure. denoted by $M_{i}$, can be estimated approximately by the

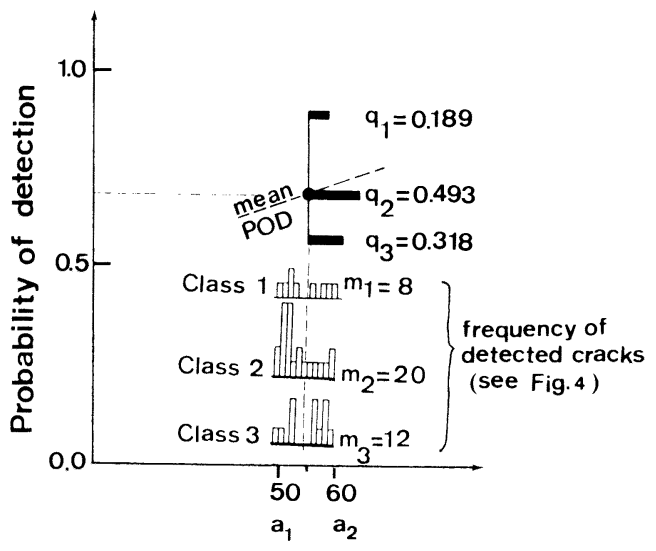

Crack length $(\mathrm{mm})$

Fig. 3 Estimation of probabilities $q_{i}$.

following equation.

$$
M_{i}=m_{i} /\left(1-\left[1-p_{i}\left\{\left(a_{1}+a_{2}\right) / 2\right\}\right]^{n}\right)
$$

where $i=1,2,3,4,5$

According to the definition, $q_{i}$ must satisfy the follow. ing two equations.

$$
\begin{aligned}
& M_{1}: M_{2}: M_{3}: M_{4}: M_{5}=q_{1}: q_{2}: q_{3}: q_{4}: q_{5} \\
& q_{1}+q_{2}+q_{3}+q_{4}+q_{5}=1.0
\end{aligned}
$$

Solving the above equations, the probabilities $q_{1}, q_{2}, \cdots$. $q_{5}$ can be determined for the cracks with lengths between $a_{1}$ and $a_{2}$. The probabilities $q_{i}$ for other crack lengths can be calculated similarly. The number of divisions of the crack lengths will be determined by the subjective judgment based on the quantity of the crack data.

\subsection{Estimation of $\boldsymbol{s}_{j}$ and $\boldsymbol{r}_{j}$}

For the false crack indications the similar method is applicable to the estimation of $s$, and $r_{j}$. Assume a false crack for which $k$ inspectors out of $n$ reported the incorrect positive indication by visual inspection. The likelihood function of this event can be written in the following form under the condition that $k>0$.

$$
Q_{m}(k / n)=\frac{{ }_{n} C_{k} s_{j}^{k}\left(1-s_{j}\right)^{n-k}}{1-\left(1-s_{j}\right)^{n}}
$$

The likelihood function for all of the false cracks of class $j$ condition is given by.

$$
\boldsymbol{L}\left(s_{j}\right)=\prod_{m=1}^{m_{r}} Q_{m}(k / n)
$$

In which $m_{F}$ is the number of false cracks belonging to class $j$ condition. Bayes' equation is applicable to the estimation of $s$.

The following three equation can be used to determine the value of $r_{j}$.

$$
\begin{aligned}
& N_{j}=m_{j} /\left\{1-\left(1-s_{j}\right)^{n}\right\} \\
& N_{1}: N_{2}: N_{3}=r_{1}: r_{2}: r_{3} \\
& r_{1}+r_{2}+r_{3}=1.0
\end{aligned}
$$

where $N_{j}$ is the number of causes plone to false indication existing in the structure, $m$; is the number of false cracks obtained by the inspection and suffix $j$ expresses class $j$ condition. 


\section{Numerical Simulation}

\section{1 Simulation method}

In order to demonstrate the validity of the proposed method, numerical simulations were carried out assuming the results of visual inspections. The contents of the inspections and the results are as follows:

Visual inspections were carried out for the same fleet structures by the same inspection team applying the same inspection method. The team consists of three inspectors and each inspector inspects the whole structure independently. The total number of locations where the positive indications were given by visual inspections is 400. Among them the correct positive indications (cracks) are 320 and the incorrect positive indications (false cracks) are 80 . In the precise inspections, the cracks were classified into three conditions, namely Class 1 (detection is easy), Class 2 (detection is moderate), and Class 3 (detection is difficult), considering all the factors except crack length. For the false cracks no classification was performed.

The contents of the 320 detected cracks and the 80 false indications are listed in Table 2 and Table 3 , respectively. Fig. 4 shows the histograms of the distri-

Table 2 Contents of the detected cracks by VI's.

\begin{tabular}{|c|c|c|c|c|c|c|c|c|c|c|c|c|}
\hline \multirow{2}{*}{$\begin{array}{c}\text { Crack } \\
\text { length } \\
\mathrm{mm}\end{array}$} & \multicolumn{4}{|c|}{$\begin{array}{c}\text { Class } 1 \\
k / n\end{array}$} & \multicolumn{4}{|c|}{$\begin{array}{c}\text { Class } 2 \\
k / n\end{array}$} & \multicolumn{4}{|c|}{$\begin{array}{c}\text { Class } 3 \\
k / n\end{array}$} \\
\hline & \begin{tabular}{|l|l} 
& 1 \\
\end{tabular} & $1 / 3$ & $2 / 3$ & $3 / 3$ & $\Sigma$ & $1 / 3$ & $2 / 3$ & $3 / 3$ & $\Sigma$ & $1 / 3$ & $2 / 3$ & $3 / 3$ \\
\hline $0.0 \_10$ & 11 & 9 & 2 & 0 & 6 & 6 & 0 & 0 & 0 & 0 & 0 & 0 \\
\hline $11 \_20$ & 13 & 9 & 4 & 0 & 11 & 8 & 3 & 0 & 2 & 2 & 0 & 0 \\
\hline 21 _30 & 12 & 3 & 5 & 4 & 19 & 13 & 4 & 2 & 4 & 4 & 0 & 0 \\
\hline $31-40$ & 6 & 1 & . & 4 & 18 & 1 & 7 & 10 & 11 & 5 & 6 & 0 \\
\hline $41 \ldots 50$ & 22 & 3 & 7 & 12 & 24 & 6 & 9 & 9 & 5 & 2 & 2 & 1 \\
\hline $51 \_60$ & 8 & 0 & 1 & 7 & 20 & 4 & 12 & 4 & 12 & 5 & 6 & 1 \\
\hline $61-70$ & 6 & 0 & 1 & 5 & 16 & 4 & 5 & 7 & 19 & 7 & 9 & 3 \\
\hline $71 \_80$ & 4 & 0 & 1 & 3 & 20 & 3 & 11 & 6 & 6 & 1 & 2 & 3 \\
\hline $81 \_90$ & 15 & 0 & 2 & 13 & 10 & 1 & 5 & 4 & 6 & 0 & 3 & 3 \\
\hline 91_100 & 3 & 1 & 0 & 2 & 6 & 1 & 5 & 0 & 5 & 0 & 3 & 2 \\
\hline$\Gamma$ & 100 & 26 & 24 & 50 & 75 & 55 & 57 & 38 & 70 & 26 & 31 & 13 \\
\hline
\end{tabular}

Table 3 Contents of the false indications.

\begin{tabular}{|c|c|}
\hline$k / n$ & $\begin{array}{c}\text { Number of False } \\
\text { Crack Indication }\end{array}$ \\
\hline $1 / 3$ & 69 \\
\hline $2 / 3$ & 11 \\
\hline $3 / 3$ & 0 \\
\hline
\end{tabular}

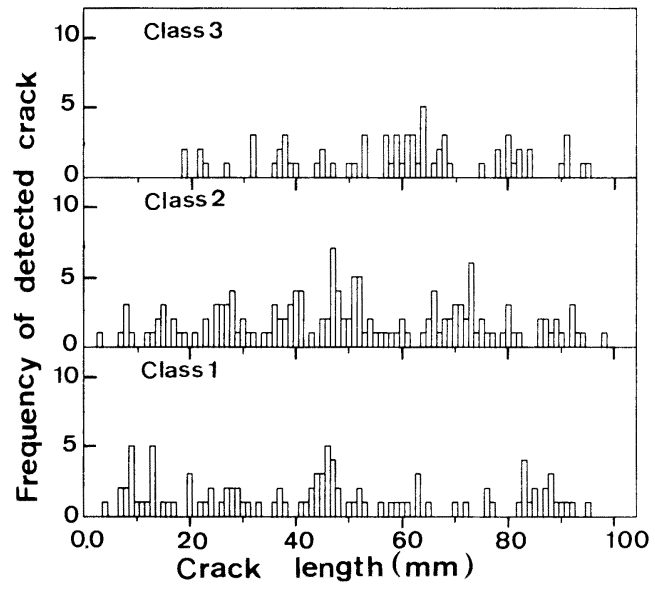

Fig. 4 Histogram of the detected crack length.

butions of crack lengths for the detected cracks of each class. The number of cracks in Class 1, Class 2 and Class 3 conditions are 100,150, and 70, respectively. Those inspection results were simulated by the Monte Carlo method, where the number of cracked locations and the distributions of crack lengths, the detection probability curves and the probability of false crack indications had been assumed for the three crack condithons.

\section{2 Results of the simulation}

Fig. 5 and Fig. 6 show the estimated $p_{i}(a)$ curves and the assumed $p_{i}(a)$ curves for the three classes, respectively. Through the comparison of these two figures, it is seen that the $p_{i}(a)$ curves can be estimated accurately by the present method.

Fig. 3 is an example of the probabilities $q_{i}$ estimated for the cracks whose lengths range between $50 \mathrm{~mm}$ and $60 \mathrm{~mm}$. The solid circle expresses the value of mean $\operatorname{POD}(a)$ at $a=55 \mathrm{~mm}$. The calculations of $q_{i}$ were carried out for each crack length at 10 -millimeter inter-

\begin{tabular}{|c|c|c|c|}
\cline { 2 - 4 } \multicolumn{1}{c|}{} & Class 1 & Class 2 & Class 3 \\
\hline$\alpha$ & -6.22 & -6.54 & -9.04 \\
\hline$\beta$ & 2.11 & 1.80 & 2.28 \\
\hline
\end{tabular}

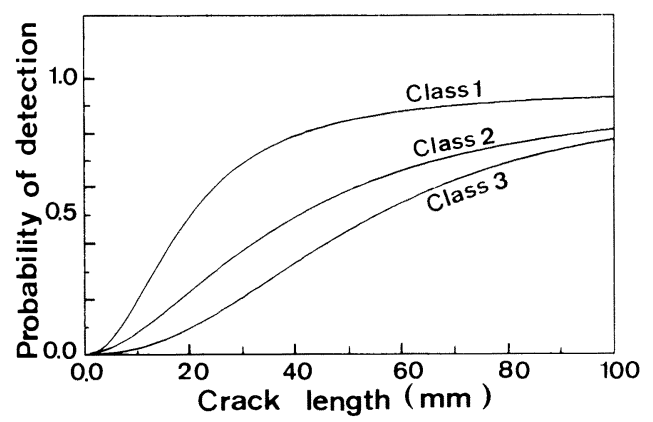

Fig. 5 Estimated $p_{i}(a)$ curves. 


\begin{tabular}{|c|c|c|c|}
\cline { 2 - 4 } \multicolumn{1}{c|}{} & Class 1 & Class 2 & Class 3 \\
\hline$\alpha$ & -8.5 & -7.0 & -9.0 \\
\hline$\beta$ & 2.5 & 1.9 & 2.2 \\
\hline
\end{tabular}

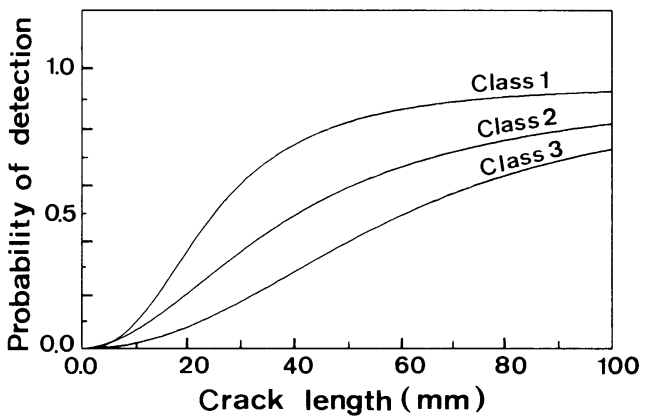

Fig. 6 Assumed $p_{i}(a)$ curves.

vals.

Fig. 7 shows the probabilities of $q_{i}$ estimated through the whole range of the crack length. The mean $\operatorname{POD}(a)$ curve which was calculated by substituting the $p_{i}(a)$ curves and probabilities $q_{i}$ into Eq. ( 1 ) is also shown in the figure. From the figure we can comprehend precisely the characteristics of the applied inspection method and the crack conditions in the structure. That is, the three $p_{i}(a)$ curves express respectively the capabilities of the inspection method for class $i$ cracks, and the heights of the histograms represent the percentages of class $i$ cracks existing in the structures.

Fig. 8 shows the distributions of the number of cracks existing in the structures estimated by Eq. ( 7 ). The

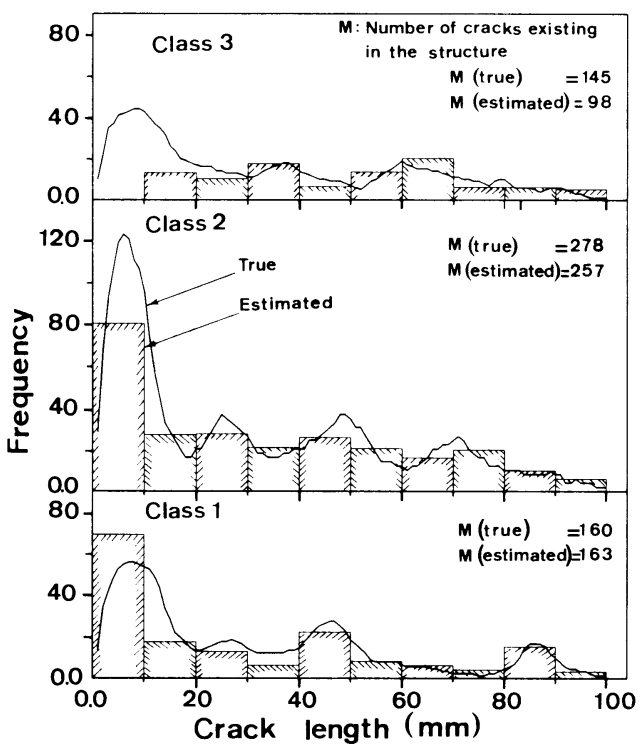

Fig. 8 Estimated and assumed distributions of crack lengths.

distributions of the true cracks assumed in the simulation are also shown in the figure. Fairly good agreement can be seen between the estimated and true distributions of the number of cracks. By using this figure. the number and the length distributions of cracks which remain after the inspection can be easily estimated.

Fig. 9 shows the posterior probability density function of the false crack indications calculated by the Bayes'

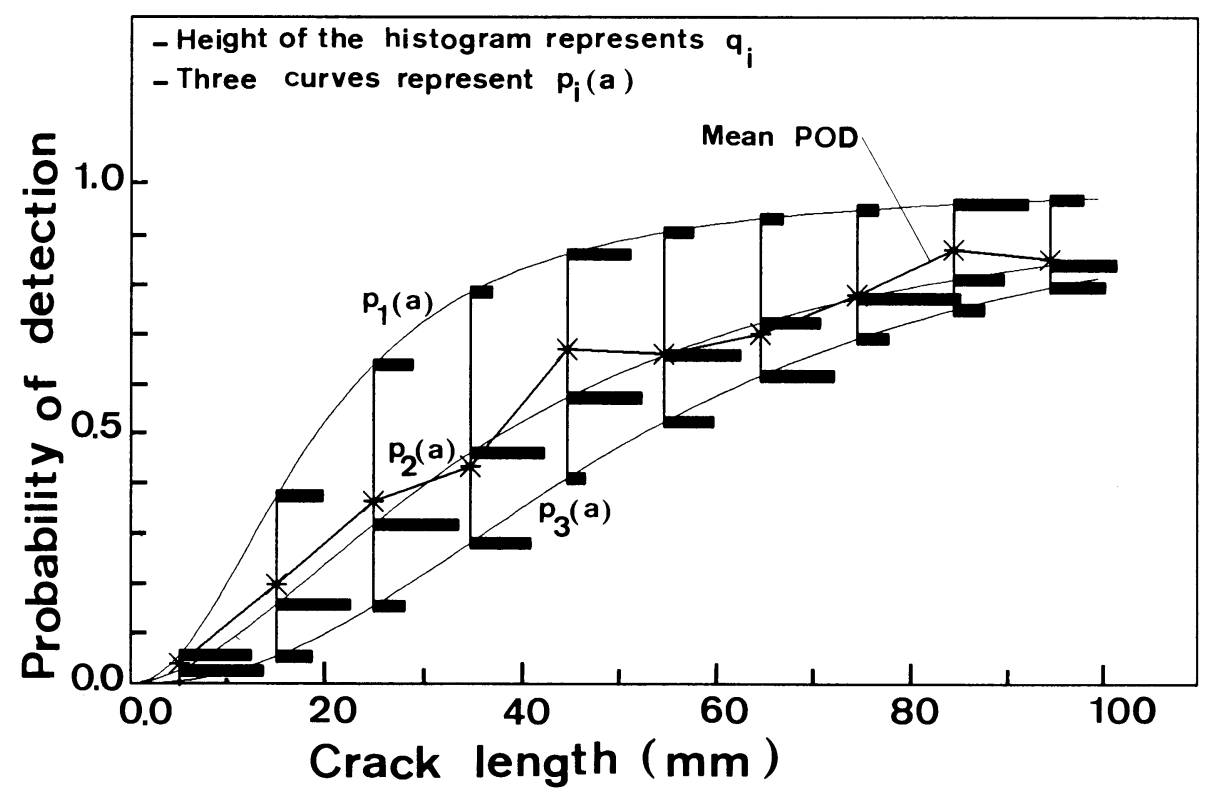

Fig. 7 Capability of applied inspection method and crack condition in the structure. 


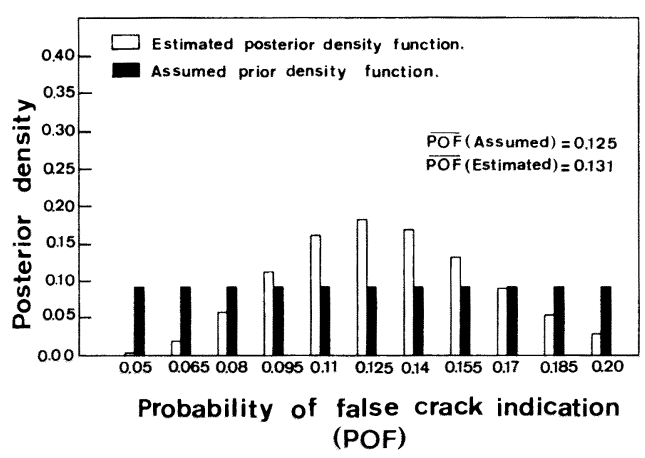

Fig. 9 Probability of false indication.

equation as well as the prior density function of that. The true value assumed as the probability of false crack indication is 0.125 . The expectation of the posterior distribution is 0.131 and this value is close to the true value.

\section{Value of Crack Classification Method}

The value of the crack classification method can be understood clearly from the following simple examples.

Example 1: Fig. 10 is a result of visual inspection carried out by three inspectors A, B and C independently for a structural model in which $M$ cracks of the same length are prepared. The number of cracks detected by each inspector is equally six, and totally ten cracks are detected.

The likelihood function of the result is given by the following equation if no classification of cracks is carried out.

$$
\begin{aligned}
\boldsymbol{L}(\mathrm{POD})= & \left\{\mathrm{POD}^{3} / Y\right\}^{2}\left\{3 \mathrm{POD}^{2}(1-\mathrm{POD}) / Y\right\}^{3} \\
& \left\{3 \mathrm{POD}(1-\mathrm{POD})^{2} / Y\right\}^{5}
\end{aligned}
$$

where

$$
Y=1-(1-\mathrm{POD})^{3}
$$

The maximum likelihood estimator of POD and $M$ are estimated as follows.

$$
\begin{aligned}
& \mathrm{POD}=0.54, \\
& M=10 /\left\{1-(1-0.54)^{3}\right\}=11.1
\end{aligned}
$$

This means that about one crack is missed by all the three inspectors (see Fig. 10(a)).

On the other hand, if the cracks are classified into three groups as shown in Fig. 10( b) from the viewpoint of detectability level, three likelihood functions are obtained. For example the likelihood function for class 3 cracks is given by

$$
\begin{aligned}
\boldsymbol{L}\left(p_{3}(a)\right)= & \left\{3 p_{3}(a)^{2}\left(1-p_{3}(a)\right) / Y\right\} \\
& \left\{3 p_{3}(a)\left(1-p_{3}(a)\right)^{2} / Y\right\}^{4}
\end{aligned}
$$

where

$$
Y=1-\left(1-p_{3}(a)\right)^{3}
$$

\begin{tabular}{|c|c|c|c|c|c|c|c|c|c|c|c|c|c|}
\hline & \multicolumn{5}{|c|}{ Detected } & \multicolumn{5}{|c|}{ cracks } & - & \multirow{2}{*}{$\begin{array}{l}\text { Missed } \\
\text { cracks }\end{array}$} \\
\hline & & 1 & 2 & 3 & 4 & 5 & 6 & 7 & 8 & 9 & 10 & 11 & \\
\hline \multirow{3}{*}{ 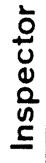 } & $A$ & $\bullet$ & $\bullet$ & 0 & $\bullet$ & $\bullet$ & 0 & • & • & 0 & 0 & 0 & \multirow{2}{*}{$\begin{array}{l}P O D=0.54 \\
M=11.1\end{array}$} \\
\hline & B & $\bullet$ & $\bullet$ & $\bullet$ & $\bullet$ & 0 & $\bullet$ & 0 & 0 & $\bullet$ & 0 & 0 & \\
\hline & C & $\bullet$ & $\bullet$ & $\bullet$ & 0 & $\bullet$ & $\bullet$ & 0 & 0 & 0 & $\bullet$ & 0 & \\
\hline
\end{tabular}

The maximum likelihood estimator is $p_{3}(a)=0.18$. Continueing the similar calculation for class 1 and class

\begin{tabular}{|c|c|c|c|c|c|c|c|c|c|c|c|c|c|c|c|c|}
\hline & \multicolumn{10}{|c|}{ Detected } & \multicolumn{5}{|c|}{ Missed cracks } \\
\hline & & 1 & 2 & 3 & 4 & 5 & 6 & 7 & 8 & 9 & 10 & $11: 12$ & 13 & 14 & 15 & 16 \\
\hline \multirow{3}{*}{ 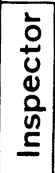 } & A & $\bullet$ & $\bullet$ & O & $\bullet$ & $\bullet$ & O & $\bullet$ & $\bullet$ & O & O & $0: 0$ & O & 0 & 0 & 0 \\
\hline & B & $\bullet$ & $\bullet$ & $\bullet$ & $\bullet$ & 0 & $\bullet$ & 0 & 0 & $\bullet$ & 0 & o & 0 & 0 & 0 & 0 \\
\hline & $\mathrm{C}$ & $\bullet$ & $\bullet$ & $\bullet$ & 0 & $\bullet$ & $\bullet$ & 0 & O & O & $\bullet$ & 00 & 0 & 0 & 0 & 0 \\
\hline
\end{tabular}
2 cracks, the mean POD and $M$ become

$$
\begin{aligned}
\mathrm{POD} & =q_{1} p_{1}(a)+q_{2} p_{2}(a)+q_{3} p_{3}(a) \\
& =0.123 * 1.0+0.193 * 0.64+0.684 * 0.18 \\
& =0.37 \\
M & =M_{1}+M_{2}+M_{3} \\
= & 2.0+3.2+11.1 \\
& =16.3
\end{aligned}
$$

This suggests that if the crack condition is good (Class

(a) No classification of cracks is applied

(b) Classification of cracks is applied

Fig. 10 Meaning of crack classification method. 
1), the detection probability is almost $100 \%$; if it is bad (Class 3 ), however, the detection probability is only $18 \%$ and about six cracks are missed by all the three inspectors.

The difference between the above two results is due to the subjective classification of cracks. Therefore, the accuracy of the estimated inspection capability can be increased if the difference of crack conditions is well reflected in the classification of cracks.

Example 2: The likelihood function of POD or $p_{i}(\alpha)$ can be used as the posterior probability density function of detection probability by adjusting the area of the likelihood distribution at unity.

Let the table in the Fig. 11 be the inspection result for a specified crack length. If no classification of cracks is carried out, the POD distribution with small variance is estimated as shown in Fig. 11 (a) according to the likelihood function. On the other hand if the classification of cracks is carried out, the POD distribution with large variance is obtained. The latter distri bution was estimated by mixing three likelihood functions for each class. This process is shown in Fig. 11 (b) in which the three distributions express $q_{1} p_{1}(a)$,

\begin{tabular}{|c|c|c|c|c|}
\hline \multicolumn{2}{|c|}{} & Class 1 & Class 2 & Class 3 \\
\hline \multirow{3}{*}{$\mathrm{k} / \mathrm{n}$} & $3 / 3$ & 5 & 4 & 2 \\
& $2 / 3$ & 4 & 4 & 4 \\
& $1 / 3$ & 0 & 1 & 2 \\
\hline \multicolumn{2}{|l|}{ Estimated $\mathrm{M}_{\mathrm{i}}$} & $\mathrm{M}_{1}=9.05$ & $\mathrm{M}_{2}=9.15$ & $\mathrm{M}_{3}=8.49$ \\
\hline
\end{tabular}
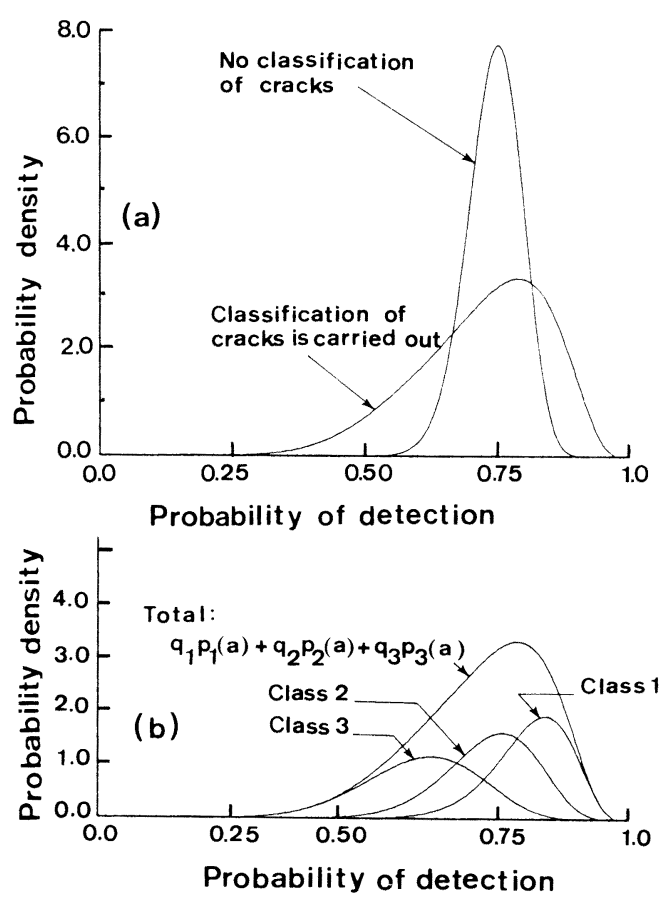

Fig. 11 Application of crack classification method to the continuous distribution of POD. $q_{2} p_{2}(a)$ and $q_{3} p_{3}(a)$, respectively. This continuous expression of POD distributions must be useful when we discuss the confidence level of POD such as 90/95 level. And the more accurate estimation of confidence level can be expected by the application of crack classification method.

\section{Applicability of the Proposed Method}

In the proposed method, the multiple visual inspections by two or more inspectors and the successive precise inspections are required to make the evaluation of the inspection capability possible. However, the multiple inspections are effective to decrease missing cracks and the precise inspections are carried out for only a limited number of locations, so this method is thought to be profitable as the inspection method of actual structures.

The following are thought to be the merits of the proposed method.

(1) The mean POD curve and the distribution property of the POD can be estimated from the records of the field inspections carried out for a structure by an inspection team under a specified inspection condition. (2) The number of cracks remaining after the inspections and the length distribution of residual cracks can be easily estimated without using any information other than inspection results.

(3) Once the detection probabilities are evaluated in the manner shown in Fig. 7, the capability of applied inspection method can be understood more precisely as compared with the ordinary mean POD curve.

When the proposed method is actually applied, further considerations must be given to the following issues :

(1) The inspectors' abilities to detect cracks have to be the same and independent for a crack. Whether these conditions are satisfied or not in the current inspections can be judged to a certain extent by careful observation of the inspection results.

(2) The difference of detectability must be well reflected in the classification of cracks, because the estimated result is influenced considerably by the classfication.

\section{Conclusion}

In this study the structural expression for the distributions of the POD and the POF in the visual inspection method has been presented, where the scatter in the distributions are caused by many factors related to the applied inspection method and the defect condition in the structure.

In order to estimate the POD and the POF properties from the results of field inspections of the structures. an inspection procedure and its analysis method has been presented. The inspection consists of the multiple visual inspections performed by two or more inspectors and the successive precise inspections carried out for 
the limited locations where positive indications were obtained by visual inspections.

From the result of the numerical simulation using an assumed inspection result in which four hundreds of correct and incorrect positive indications obtained by the three inspectors were prepared, it is concluded that the characteristics of the distribution of POD as well as POF can be estimated accurately by the proposed method.

\section{References}

1) Yang, J-N. and Trapp, W. J. : Reliability Analy sis of Aircraft structures Under Random Loading and Periodic Inspection, AIAA Journal. Vol. 12. No. 12, pp. 1623, December (1974).

2) Itagaki, H., Hiraoka, K., Asada, H. and Itoh, S. : Reliability Analysis of Aircraft Structures With Multiple Site Fatigue Cracks, JCOSSAR'87, Vol. 1. pp. 147-151 (1987) (in Japanese)

3) Fujimoto, Y., Ideguchi, A. and Iwata, M. : Reliability Assessment for Deteriorating Structure by Markov Chain Model, Journal of the Society of Naval Architects of Japan, Vol. 166, pp. 303-314 (1989) (in Japanese)

4) Berens, A. P. and Hovey, P. W.: Statistical
Methods for Estimating Crack Detection Probabilities, ASTM, STP-798, pp. 79-94 (1983).

5) Arakawa, T.: The Recent Trend of Investigation on Ability of Defect Detection by NonDestructive Examinations, Journal of the Japan Welding Society, Vol. 55, No. 4, pp. 35-42 (1986) (in Japanese).

6) Satoh, K, Toyoda, M., Minami, F. Fujimori, T., and Nakatsuji, T.: Reliability of Inspected Welds Taking Defect Detectability of NDT into Account and Influence Factors to Reliability of Welds, Journal of the Japan Welding Society, Vol. 50, No. 1, pp. 47-52 (1981) (in Japanese).

7) Goranson, U. G., and Rogers, T.: Elements of Damage Tolerance Verification, 12th Symposium of the International Commitee on Aeronautical Fatigue (ICAF) (1983).

8) Frieze, P. A., and Kam, J. C.: Reliability Assessment of the Non-Destructive Inspection on Offshore Tubular Structures, Eighth Int. Conf. on Offshore Mechancs and Arctic Engineering (OMAE), pp. 733-739 (1989).

9) Itagaki, H., Ozaki, F. and Nemoto, T.: On the Estimation of the Probability Distribution Func. tion of Defects in Structure, Jour. of the Society of Naval Architects of Japan, Vol. 139, pp. 292301 (1976) (in Japanese). 